\title{
ON GELFAND-MAZUR THEOREM
}

\section{GIUSEPPE IURATO}

Department of Physics and Chemistry

University of Palermo

Italy

e-mail: giuseppe.iurato@unipa.it

\begin{abstract}
From a suitable extension of the notion of spectrum drew from normed algebra theory, it will be possible, among other things, to provide some generalizations of the well-known Gelfand-Mazur theorem. In this brief research report, we wish to pursue one of these, as achieved in $\mathbf{I , 4}$.
\end{abstract}

\section{Introduction}

Let $\mathbb{K}$ be an arbitrary field of characteristic zero ${ }^{1}$, not necessarily algebraically closed.

Let $A_{\mathbb{K}}$ be an arbitrary linear unitary commutative $\mathbb{K}$-algebra and $G\left(A_{\mathbb{K}}\right)$ be the group of units of $A_{\mathbb{K}}$. For each $a \in A_{\mathbb{K}}$, let ${ }^{2}$

$$
\sigma_{A_{\mathbb{K}}, \mathbb{K}}(a) \doteq\left\{\lambda ; \lambda \in \mathbb{K} \text { such that } \nexists\left(a-\lambda 1_{A_{\mathbb{K}}}\right)^{-1}\right\}
$$

${ }^{1}$ Whence card $\mathbb{K}=\infty$.

${ }^{2} 1_{A_{\mathbb{K}}}$ denotes the unit of such a $\mathbb{K}$-algebra.

2010 Mathematics Subject Classification: 13J99, 46H05, 46H30.

Keywords and phrases: spectrum, Gelfand-Mazur theorem, topology.

Received June 26, 2015

(C) 2015 Scientific Advances Publishers 
that we say to be the $\left(A_{\mathbb{K}}, \mathbb{K}\right)$-spectrum of $a$; $r_{A_{\mathbb{K}}, \mathbb{K}}(a) \doteq \mathbb{K} \backslash \sigma_{A_{\mathbb{K}}, \mathbb{K}}(a)$ is said to be the $\left(A_{\mathbb{K}}, \mathbb{K}\right)$-resolvent of $a$.

There exist linear unitary commutative $\mathbb{K}$-algebras in which such a spectrum may be empty for certain their elements: for instance, if $A_{\mathbb{K}}$ is a linear unitary commutative integral $\mathbb{K}$-algebra of finite degree $(>1)$ over $\mathbb{K}$, then it follows that it is a field (because $\varphi_{a}: x \rightarrow a x \forall x \in A_{\mathbb{K}}$ is an automorphism of $A_{\mathbb{K}}$, for each $a \in A_{\mathbb{K}}$ arbitrarily fixed), so that $\sigma_{A_{\mathbb{K}}, \mathbb{K}}(a)=\emptyset$ for each $a \in A_{\mathbb{K}} \backslash \mathbb{K} \mathbb{A}_{\mathbb{K}}(\neq \emptyset)$ because, being $a-\lambda 1_{A_{\mathbb{K}}} \in A_{\mathbb{K}} \backslash\{0\} \forall \lambda \in \mathbb{K}$, there always exists $\left(a-\lambda 1_{A_{\mathbb{K}}}\right)^{-1}$.

Likewise, if $A_{\mathbb{K}}=\mathbb{K}(X)$, then $\sigma_{A_{\mathbb{K}}, \mathbb{K}}(X)=\emptyset$.

It follows that the question related to the emptiness or not, of the spectrum of the generic element of a given linear unitary commutative $\mathbb{K}$-algebra, is not trivial.

For the first elementary properties of the spectrum, we refer to [1] and [3].

Let $\mathbb{K}$ be an arbitrary field. If $A_{\mathbb{K}}$ is an arbitrary linear unitary commutative $\mathbb{K}$-algebra such that $A_{\mathbb{K}} / I \cong \mathbb{K}$ for every maximal ideal $I$ of $A_{\mathbb{K}}$, then $A_{\mathbb{K}}$ is said to be a spectral algebra (see [10, Chapter 2, Section 1]). So, for instance, the Weak Nullstellensatz proves, amongst other, that $\mathbb{K}\left[X_{1}, \ldots, X_{n}\right]$ is a spectral algebra when $\mathbb{K}$ is algebraically closed (see [1], where a new alternative proof of Weak Nullstellenstaz making use of the above notion of spectrum, is provided).

Let $\mathbb{K}$ be an arbitrary field of characteristic zero. We remember that $A_{\mathbb{K}}$ is always a $\mathbb{K}$-linear space, so that we set (with abuse of notation) $\left[A_{\mathbb{K}}: \mathbb{K}\right]=\operatorname{dim}_{\mathbb{K}} A_{\mathbb{K}}$. Furthermore, if every non-zero element of $A_{\mathbb{K}}$ has a multiplicative inverse (that is, $A_{\mathbb{K}}$ is a field extension of $\mathbb{K}$ ), then we call $A_{\mathbb{K}}$, more specifically, a division algebra (according to Van der Waerden - see [5]). 
I. If $A_{\mathbb{K}}$ is a linear unitary commutative integral $\mathbb{K}$-algebra, and $\mathbb{K}$ is an arbitrary field of characteristic zero, then we have the following results:

1. If $\mathbb{K}$ is not algebraically closed and $2 \leq\left[A_{\mathbb{K}}: \mathbb{K}\right]<\infty$, then there exists, at least, one $a \in A_{\mathbb{K}}$ such that $\sigma_{A_{\mathbb{K}}, \mathbb{K}}(a)=\emptyset$ (see final Note 1 ).

2. If $\left[A_{\mathbb{K}}: \mathbb{K}\right]=\infty, \mathbb{K}$ is not algebraically closed and $A_{\mathbb{K}}$ is a division algebra, then there exists, at least, one $a \in A_{\mathbb{K}}$ such that $\sigma_{A_{\mathbb{K}}, \mathbb{K}}(a)=\emptyset$ (see final Note 1).

3. If $\left[A_{\mathbb{K}}: \mathbb{K}\right]=\infty$ and $A_{\mathbb{K}}$ is not a division algebra (hence not finitely generated as a $\mathbb{K}$-algebra ${ }^{3}$ ), then $\sigma_{A_{\mathbb{K}}, \mathbb{K}}(a) \neq \emptyset$ for each $a \in A_{\mathbb{K}}$, whatever be $\mathbb{K}$ (see final Note 1).

4. (Generalized Gelfand-Mazur) If $\left[A_{\mathbb{K}}: \mathbb{K}\right]=1$, then $\sigma_{A_{\mathbb{K}}, \mathbb{K}}(a)=\emptyset$ for each $a \in A_{\mathbb{K}}$, and $A_{\mathbb{K}} \cong \mathbb{K}$, whatever be $\mathbb{K}$ (see Remark 1 ).

If $A_{\mathbb{K}}$ is a linear unitary commutative $\mathbb{K}$-algebra, $\mathbb{K}$ is an algebraically closed field and $\left[A_{\mathbb{K}}: \mathbb{K}\right]<\operatorname{card} \mathbb{K}$, then $A_{\mathbb{K}}$ is a spectral algebra, and therefore $\sigma_{A_{\mathbb{K}}, \mathbb{K}}(a)=\emptyset$ for each $a \in A_{\mathbb{K}}$.

Let us prove the first part of the theorem given by $1,2,3$, and 4 .

If $2 \leq\left[A_{\mathbb{K}}: \mathbb{K}\right]<\infty$, since $A_{\mathbb{K}}$ is an integral domain and a finite dimensional $\mathbb{K}$-linear space, it follows that it is a field (because $\varphi_{a}: x \rightarrow a x \forall x \in A_{\mathbb{K}}$ is an automorphism for every nonzero $a \in A$ ),

${ }^{3}$ Therefore, taking into account 2 , if $\mathbb{K}$ is not algebraically closed, then $A_{\mathbb{K}}$ may be a division algebra, finitely generated as a $\mathbb{K}$-algebra. For instance, $\mathbb{K}(X)$ is a division algebra (finitely generated field extension of $\mathbb{K}$ ), not finitely generated as a $\mathbb{K}$-algebra, for which 3 . does not hold, as we have already seen. See also next Note 1. 
with $A_{\mathbb{K}} \backslash \mathbb{K} 1_{A_{\mathbb{K}}} \neq \emptyset \quad\left(\right.$ by $\left[A_{\mathbb{K}}: \mathbb{K}\right] \geq 2$ ), so, for each $a \in A_{\mathbb{K}} \backslash \mathbb{K} 1_{A_{\mathbb{K}}}$, we have that there exists $\left(a-\lambda 1_{A_{\mathbb{K}}}\right)^{-1} \in A_{\mathbb{K}} \backslash \mathbb{K} 1_{A_{\mathbb{K}}} \forall \lambda \in \mathbb{K}$; thus $\sigma_{A_{\mathbb{K}}, \mathbb{K}}(a)=\emptyset$. Therefore, (1) is proved ${ }^{4}$.

Since $A_{\mathbb{K}}$ is a division algebra, we may suppose it is a field. Furthermore, since $\left[A_{\mathbb{K}}: \mathbb{K}\right]=\infty$ and $\mathbb{K}$ is not algebraically closed, it follows that $A_{\mathbb{K}}$ is a proper field extension of $\mathbb{K}$, so $A_{\mathbb{K}} \backslash \mathbb{K} 1_{A_{\mathbb{K}}} \neq \emptyset$, and, hence, there exists $\left(a-\lambda 1_{A_{\mathbb{K}}}\right)^{-1} \in A_{\mathbb{K}} \backslash\left\{0_{A_{\mathbb{K}}}\right\} \forall \lambda \in \mathbb{K} \quad$ and $\forall a \in A_{\mathbb{K}} \backslash \mathbb{K} 1_{A_{\mathbb{K}}}$, that is, $\sigma_{A_{\mathbb{K}}, \mathbb{K}}(a)=\emptyset$ for every $a \in A_{\mathbb{K}} \backslash \mathbb{K} 1_{A_{\mathbb{K}}}$, whence 5 .

If $\left[A_{\mathbb{K}}: \mathbb{K}\right]=1$, then $A_{\mathbb{K}}$ is a trivial extension of $\mathbb{K}$, so that $\sigma_{A_{\mathbb{K}}, \mathbb{K}}(a)=\left\{\lambda_{a}\right\}$ with $a=\lambda_{a} 1_{A_{\mathbb{K}}}$ for a unique $\lambda_{a} \in \mathbb{K}$, for every $a \in A_{\mathbb{K}}$ arbitrarily fixed; hence, $\sigma_{A_{\mathbb{K}}, \mathbb{K}}(a) \neq \emptyset \forall a \in A_{\mathbb{K}}$, and thus 4 . is proved, with $A_{\mathbb{K}} \stackrel{⿱}{\cong} \mathbb{K}$, where $\psi: a \rightarrow \lambda_{a} \forall a \in A_{\mathbb{K}}$.

To prove 3, we assume, by contradiction, it is not true. Let $\sigma_{A_{\mathbb{K}}, \mathbb{K}}(a)=\emptyset$ for, at least, one $a \in A_{\mathbb{K}}$, so that we have $\exists\left(a-\lambda 1_{A_{\mathbb{K}}}\right)^{-1}$ $\forall \lambda \in \mathbb{K}$, whence it follows that, in particular, $a \in G\left(A_{\mathbb{K}}\right)$. If $a=1_{A_{\mathbb{K}}}$, then for $\lambda=1_{\mathbb{K}}$, we reach an absurdity so that let $a \in G\left(A_{\mathbb{K}}\right) \backslash\left\{1_{A_{\mathbb{K}}}\right\}$. If $G\left(A_{\mathbb{K}}\right)=\left\{1_{A_{\mathbb{K}}}\right\}$, then we get again an absurdity, so we suppose $\emptyset \neq G\left(A_{\mathbb{K}}\right) \backslash\left\{1_{A_{\mathbb{K}}}\right\} \subset A_{\mathbb{K}}$, being $G\left(A_{\mathbb{K}}\right) \backslash\left\{1_{A_{\mathbb{K}}}\right\} \neq A_{\mathbb{K}}$ because $A_{\mathbb{K}}$ is not a division algebra. Therefore, let $a \in G\left(A_{\mathbb{K}}\right) \backslash\left\{1_{A_{\mathbb{K}}}\right\}$.

\footnotetext{
${ }^{4}$ For instance, if $A_{\mathbb{K}}=\mathbb{C}_{\mathbb{R}}$, then $\sigma_{\mathbb{C}_{\mathbb{R}}, \mathbb{R}}(i)=\emptyset$.

${ }^{5}$ For instance, if $A_{\mathbb{K}}=\mathbb{R}_{Q}$, then $[\mathbb{R}: \mathbb{Q}]=\infty$ and $\sigma_{\mathbb{R}}, \mathbb{Q}(e)=\emptyset$.
} 
We now consider $A[[\lambda]]$, i.e., the linear unitary commutative integral $\mathbb{K}$-algebra of the formal power series in the variable $\lambda$ (considered, a priori, as an abstract theoretical variable), with coefficients in $A$ (= support of $\left.A_{\mathbb{K}}\right)$. Since $a \in G\left(A_{\mathbb{K}}\right) \backslash\left\{1_{A_{\mathbb{K}}}\right\}$, we have 6

$$
\left(a-\lambda 1_{A_{\mathbb{K}}}\right)^{-1}=\sum_{n \in \mathbb{N}_{0}} a^{-(n+1)} \lambda^{n}, \quad\left(a^{-1}-\lambda 1_{A_{\mathbb{K}}}\right)^{-1}=\sum_{n \in \mathbb{N}_{0}} a^{n+1} \lambda^{n} .
$$

We endow $A[[\lambda]]$ with a suitable topology as follows. We recall that, if $\Omega$ is an abstract set (of indices), and $A$ is a unitary commutative ring, then we denote the total algebra, generated by the monoid (of multi-indices) $\mathbb{N}_{0}^{\Omega}$ over $A$, by $A\left[\left[\left(X_{i}\right)_{i \in \Omega}\right]\right]$ : it is the algebra of formal power series in the indeterminates $X_{i}(i \in \Omega)$ with coefficients in $A$, whose generic element (in multi-index notation) is of the type $u=\sum_{\nu \in \mathbb{N}_{0}^{\Omega}} a_{\nu} X^{\nu}$ with general term $a_{\nu} X^{\nu}$ of degree $|\nu| \geq 0$. By the well-known identification series-sequences, we have $A\left[\left[\left(X_{i}\right)_{i \in \Omega}\right]\right] \cong A^{\mathbb{N}_{0}^{\Omega}}$, where $A^{\mathbb{N}_{0}^{\Omega}}$ is the formal Cartesian product of many copies of $A$, that is, of the type

${ }^{6}$ In a power series ring $A[[T]]$, where $A$ is an arbitrary unitary commutative ring, the following Neumann's expansion $(1-T)^{-1}=\sum_{n \in \mathbb{N}_{0}} T^{n}$ subsists. Moreover, we recall that, if one considers the norm

$$
\nu: A[[\lambda]] \rightarrow \mathbb{N}_{0}, \quad \nu\left(\sum_{n \in \mathbb{N}_{0}} a_{n} \lambda^{n}\right) \doteq \ll \text { order of } \sum_{n \in \mathbb{N}_{0}} a_{n} \lambda^{n} »,
$$

then it is possible to define the following convergence criterion:

$$
\sum_{n \in \mathbb{N}_{0}} a_{n} \lambda^{n}=\lim _{n \rightarrow \infty} \sum_{i=0}^{n} a_{i} \lambda^{i} \stackrel{\text { def }}{\Leftrightarrow} \nu\left(\sum_{n \in \mathbb{N}_{0}} a_{n} \lambda^{n}-\sum_{i=0}^{n} a_{i} \lambda^{i}\right) \geq n \text { definitively }
$$

whence the following result arises: if $\left\{\chi_{n}\right\}_{n \in \mathbb{N}_{0}}$ is a sequence of $A[[\lambda]]$, then $\sum_{n \in \mathbb{N}_{0}} \chi_{n}$ converges if and only if $\lim _{n \rightarrow \infty} \nu\left(\chi_{n}\right)=+\infty$, whence, by Equation (2), it also follows that the general term of a convergent power series tends to zero (see [6, Capítulo 1, Seccíon 1.5], [2] and the next proposition II, 2). 


$$
\prod_{i \in I} S_{i} \doteq\left\{\varphi ; \varphi: I \rightarrow \bigcup_{i \in I} S_{i}, \varphi(i) \in S_{i} \forall i \in I\right\},
$$

where $I=\mathbb{N}_{0}^{\Omega}$ and $S_{i}=A \forall i$. By the Axiom of Choice (Zermelo) $A^{\mathbb{N}_{0}^{\Omega}} \neq \emptyset$. Hence, for instance, it is possible to endow $A$ with the discrete topology, and then to equip $\left(A^{\mathbb{N}_{0}^{\Omega}} \cong\right) A\left[\left[\left(X_{i}\right)_{i \in \Omega}\right]\right]$ with the product topology which we will call the canonical topology; if card $\Omega<\infty$, then such a topology is discrete. In this topological space, it is possible to prove the following lemma (see [2, Chapter IV, Section 4, n. 2] and references therein).

II. Let $\Xi$ be an infinite set of indices and $\left(u_{j}\right)_{j \in \Omega}$ be a family of elements of $A\left[\left[\left(X_{i}\right)_{i \in \Omega}\right]\right]$ with $u_{j}=\sum_{\nu} a_{j \nu} X^{\nu}$ for each $j \in \Xi$. Then, the following conditions are equivalent amongst them:

1. The family $\left(u_{j}\right)_{j \in \Xi}$ is summable in $A\left[\left[\left(X_{i}\right)_{i \in \Omega}\right]\right]$.

2. We have $\lim _{j} u_{j}=0$ taken along the filter of complements of finite subsets of $\Xi$ (cofinite of Fréchet filter ${ }^{7}$ ).

3. For every $\nu \in \mathbb{N}_{0}^{\Omega}$, we have $a_{j v}=0$ except for a finite number of indices $j \in \Xi$. When, at least, one of these conditions holds, then the series $u=\sum_{j \in \Xi} u_{j}$ is equal to $\sum_{\nu} a_{\nu} X^{\nu}$ with $a_{\nu}=\sum_{j \in \Xi} a_{j \nu}$ for each $\nu \in \mathbb{N}_{0}^{\Omega}$

Remark. As an example of summable family, we consider the following: if $u \in A\left[\left[\left(X_{i}\right)_{i \in \Omega}\right]\right]$ with $a_{\nu}$ the coefficient of $X^{\nu}$ in $u$, then the family $\left(a_{\nu} X^{\nu}\right)_{\nu \in \mathbb{N}_{0}^{\Omega}}$ is summable with sum $u$ (see, also, footnote 6).

${ }^{7}$ See also what has been said in the last part of the previous footnote 6 . 
In our case, we consider $A[[\lambda]]$ as above with card $\Omega=1$ (hence, with the discrete canonical topology), so that $\left(X_{i}\right)_{i \in \Omega}$ reduces only to $\lambda$. By the convergence of the series of (1) for every $\lambda \in \mathbb{K}$ (as well as taking into account what is said in footnote ${ }^{6}$ ), it follows that the general term of these, tends to 0 in the cofinite topology of ${ }^{6}$ II, 2 , whence it also follows that

$$
\lim _{n \rightarrow \infty} a^{-(n+1)} \lambda^{n}=\lim _{n \rightarrow \infty} a^{(n+1)} \lambda^{n}=0
$$

in $\mathbb{N}_{0}$. On the other hand, card $\mathbb{K}=\infty$ because $\mathbb{K}$ has characteristic zero, so that it is also card $A=\infty$, and hence there is a natural continuous $^{8}$ bijection (see [3]), say $\Psi: A[\lambda] \rightarrow \mathcal{F}_{\text {pol }}(A, A)$, between the algebra of (abstract) polynomials $A[\lambda]$ (which is a dense subset of $A[[\lambda]]$, so that $\Psi$ can be, in a unique manner, continuously extended to the whole of $A[[\lambda]])$ and the algebra of polynomial functions $\mathcal{F}_{\text {pol }}(A, A)$ (with elements $f: \lambda \rightarrow f(\lambda) \in A \forall \lambda \in A$ ). Therefore, the relations (9) in $A[\lambda]$, hold too in $\mathcal{F}_{p o l}(A, A)$ vias $\Psi$, so that we have $a^{-(n+1)} \lambda^{n} \rightarrow 0$ and $a^{(n+1)} \lambda^{n} \rightarrow 0$ for every $\lambda \in A$, hence also for $\lambda=1_{A}$, whence

${ }^{8}$ This continuity condition surely holds, whatever is the topology given in $\mathcal{F}_{\text {pol }}(A, A)$ provided that it is at least of Hausdorff type, because we have supposed $A[[\lambda]]$ equipped with the discrete canonical topology (since card $\Omega=1$ ), so that the related induced topology on $A[\lambda]$ is also discrete. Furthermore, since we have supposed that $A$ is neither a finitely generated algebra (as a $\mathbb{K}$-algebra) nor a division algebra, the natural identification $A[\lambda] \stackrel{\Psi}{\cong} \mathcal{F}_{\text {pol }}(A, A)$ does not have strange pathological cases: for example, it does not subsist when $A$ is the (infinite) pure transcendental field $\mathbb{K}(X)$, since, due to the singularities of the rational functions, these latter cannot be extended, as functions, upon the whole of $A$. Finally, under these hypotheses, thanks to the continuity of the bijection $\Psi$, we have that $x_{n} \rightarrow x$ implies $\Psi\left(x_{n}\right) \rightarrow \Psi(x)$, for every $\left\{x_{n}\right\}_{n \in \mathbb{N}}$ in $A[[\lambda]]$. Indeed, if $x_{n} \rightarrow x$, then, for each open neighbourhood $U(\Psi(x))$ of $\Psi(x), \Psi^{-1}(U(\Psi(x)))$ is an open neighbourhood of $x=\Psi^{-1}(\Psi(x))$ because of the continuity of $\Psi$, so that $x_{n} \in \Psi^{-1}(U(\Psi(x)))$ definitively, hence $\Psi\left(x_{n}\right) \in \Psi\left(\Psi^{-1}(U(\Psi(x)))\right)=U(\Psi(x))$ definitively, whence $\Psi\left(x_{n}\right) \rightarrow \Psi(x)$. 


$$
\lim _{n \rightarrow \infty} a^{-(n+1)}=0, \quad \lim _{n \rightarrow \infty} a^{(n+1)}=0 .
$$

But, since $\lambda$ is arbitrary, for every $\lambda \neq 0$, we have the identity

$$
\left(a^{-1}-\lambda 1_{A}\right)^{-1}=-\lambda^{-1} a\left(a-\lambda^{-1} 1_{A}\right)^{-1} \quad \forall \lambda \in \mathbb{K} \backslash\{0\},
$$

from which it follows that (10) must hold simultaneously, and this is an absurdity since (in the integral domain $A$ ) we have $a^{-(n+1)} a^{(n+1)}=1_{A}$ $\forall n \in \mathbb{N}_{0}$. Hence, 3. must be true.

We finally prove the second and last part of the theorem.

First of all, since char $\mathbb{K}=0$, we have card $\mathbb{K}=\infty$, whence $\operatorname{card} A_{\mathbb{K}}=\infty$ (independently by $\left[A_{\mathbb{K}}: \mathbb{K}\right]<\operatorname{card} \mathbb{K}=\infty$ ).

If $I$ is an arbitrary maximal ideal of $A_{\mathbb{K}}$, then it follows that $\widetilde{A}_{\mathbb{K}}=$ $A_{\mathbb{K}} / I$ is a field extension of $\mathbb{K}$. We suppose, by contrast, that $\widetilde{A}_{\mathbb{K}} \neq \mathbb{K}$, so, being $\widetilde{A}_{\mathbb{K}}$ a field extension of $\mathbb{K}$, in any case we have $\mathbb{K} \subseteq \widetilde{A}_{\mathbb{K}}$, so that, let $\bar{x} \in \widetilde{A}_{\mathbb{K}} \backslash \mathbb{K}(\neq \emptyset)$. Hence $\left(\bar{x}-\lambda 1_{\widetilde{A}_{\mathbb{K}}}\right)$ is invertible for every $\lambda \in \mathbb{K}$, that is, $\quad \sigma_{\widetilde{A}_{\mathbb{K}}, \mathbb{K}}(\bar{x})=\emptyset$, and therefore $\rho_{\widetilde{A}_{\mathbb{K}}, \mathbb{K}}(\bar{x})=\mathbb{K}$. Then, since $n=\left[A_{\mathbb{K}}: \mathbb{K}\right]<$ card $\mathbb{K}=\infty$, if $\left\{x_{1}, \ldots, x_{n}\right\}$ is a system of generators for $A_{\mathbb{K}}$, it follows that $\left\{\tilde{x}=x_{i}+I ; i=1, \ldots, n\right\}$ is a system of generators for $\widetilde{A}_{\mathbb{K}}$, so that (by Steiner's lemma of linear algebra) $\left[\widetilde{A}_{\mathbb{K}}: \mathbb{K}\right] \leq n<\operatorname{card} \mathbb{K}$. Hence, $\left\{\left(\tilde{x}-\lambda 1_{\tilde{A}_{\mathbb{K}}}\right)^{-1} ; \lambda \in \mathbb{K}\right\}$ is necessarily a linearly dependent system because we have $\operatorname{card}\left\{\left(\tilde{x}-\lambda 1_{\widetilde{A}_{\mathbb{K}}}\right)^{-1} ; \lambda \in \mathbb{K}\right\}=\operatorname{card} \rho_{\widetilde{A}_{\mathbb{K}}, \mathbb{K}}(\bar{x})=\operatorname{card} \mathbb{K}$, whence

$$
\sum_{i \in \Lambda} \mu_{i}\left(\tilde{x}-\lambda_{i} 1_{\tilde{A}_{\mathbb{K}}}\right)^{-1}=0 \widetilde{A}_{\mathbb{K}} \text { for some } \mu_{i} \in \mathbb{K}, i \in \Lambda \text {, not all zero, }
$$

and for each finite set (of indices) $\Lambda$. Therefore, $\bar{x}$ is algebraic over $\mathbb{K}$, so that $\bar{x} \in \mathbb{K}$ (being $\mathbb{K}$ algebraically closed), which is impossible. Thus $\widetilde{A}_{\mathbb{K}} \cong \mathbb{K}$, and we shall obtain the complete proof arguing as in [1, IV]. 
Note 1. Under the hypotheses of $\mathbf{I}$, if $\mathbb{K}$ is algebraically closed, then $2 \leq\left[A_{\mathbb{K}}: \mathbb{K}\right]<\infty$ cannot be true. In fact, since $A_{\mathbb{K}}$ is an integral domain, if it were $1<\left[A_{\mathbb{K}}: \mathbb{K}\right]<\infty$, then $A_{\mathbb{K}}$ would be a field (as proved in 1.), hence a finite degree field extension of $\mathbb{K}$ (which is necessarily algebraic - see [2]) and, therefore, it should be $A_{\mathbb{K}} \cong \mathbb{K}$ because $\mathbb{K}$ is algebraically closed. Hence $\operatorname{dim}_{\mathbb{K}} A_{\mathbb{K}}=1$, against $\left[A_{\mathbb{K}}: \mathbb{K}\right]>1$.

Likewise, ever due to the algebraic closure of $\mathbb{K}$, we obtain an absurdity if we suppose $\left[A_{\mathbb{K}}: \mathbb{K}\right]=\infty, \mathbb{K}$ algebraically closed and $A_{\mathbb{K}}$ a division algebra, whereas, if $\mathbb{K}$ is algebraically closed and $A_{\mathbb{K}}$ is a division algebra, then (see $\mathbf{I}, 4)$ it has to be $A_{\mathbb{K}} \cong \mathbb{K}$, and so, in this case, we have again $\sigma_{A_{\mathbb{K}}}, \mathbb{K}(a) \neq \emptyset \forall a \in A_{\mathbb{K}}$. The proof of 3 , has been conducted through an auxiliary artifice, precisely considering $A[[\lambda]]$ equipped with a particular product topology (the canonical one, which is discrete for card $\Omega=1$ ) and $\mathcal{F}_{\text {pof }}(A, A)$ endowed with an arbitrary Hausdorff topology.

Finally, we observe that the condition $\left[A_{\mathbb{K}}: \mathbb{K}\right]<$ card $\mathbb{K}$, of the last part of the theorem $\mathbf{I}$, is satisfied by every linear unitary commutative $\mathbb{K}$-algebra having a (finite or) infinite system of generators $\Theta$ whose cardinality has an infinity's order less than of $\mathbb{K}$ (we recall that card $\mathbb{K}=\infty$, since $\mathbb{K}$ is algebraically closed): for instance, it may be card $\Theta=\aleph_{0}$ and card $\mathbb{K}=2 \aleph_{0}$.

Then, if we keep into account the infinity's order of card $\mathbb{K}$, it is possible to opportunely extend the first part of $\mathbf{I}$, on the basis of its second part.

Remark 1. Historically, the origin of the notion of spectrum of an element of an algebra, may be partially recognized in a proof given by Weyl in [5, Chapter V, Part A, Section 7, p. 316] (of the Dover edition), where it is proved that the only division algebra of finite order, over an algebraically closed field, is this field itself. 
On the other hand, the so-called Gelfand-Mazur theorem states that every complex Banach algebra - that is, a division algebra - is (isometrically) isomorphic to $\mathbb{C}$. This theorem is truly one of the most fundamental theorems in the study of commutative Banach algebras: it was announced by Mazur in 1938 (see [7]) and proved by Gelfand in 1941 (see [8]). For various other proofs of this theorem, see [9].

\section{References}

[1] G. Iurato, A note on Hilbert's weak Nullstellensatz, International Journal of Advances in Applied Mathematics and Mechanics 2(4) (2015), 18-24.

[2] N. Bourbaki, Algebra II, Springer-Verlag, Berlin and Heidelberg, 1990.

[3] I. M. Gelfand, D. Raikov and G. Shilov, Commutative Normed Rings, Chelsea Publishing Comp., New York, 1964.

[4] M. Girardi and G. Israel, Teoria dei campi, Giangiacomo Feltrinelli Editore, Milano, 1976.

[5] H. Weyl, Gruppentheorie und Quantenmechanik, Verlag Von S. Hirzel, Leipzig, 1928 (English Translation by H.P. Robertson, The Theory of Groups and Quantum Mechanics, Methuen, London, 1931, reprinted by Dover Publications, Inc., New York, 1950).

[6] C. I. Castillo, Geometría Algebraica, Departamento de Matemáticas parala Economía y la Empresa, Facultad de Economía, Universidad de Valencia, Valencia, 2008.

[7] S. Mazur, Sur les Anneaux Lineaires, C. R. Acad. Sci. Paris 207 (1938), 1025-1027.

[8] I. M. Gelfand, Normierte Ringe, Mat. Sbornik 9(51) (1941), 3-24.

[9] C. E. Rickart, General Theory of Banach Algebras, David Van Nostrand Company, Inc., Princeton, New Jersey, 1960.

[10] Yu. I. Lyubich, Functional Analysis I: Linear Functional Analysis, in: N. K. Nikol'skij (Ed.), Functional Analysis, Encyclopaedia of Mathematical Sciences, Editor-in-Chief: R. V. Gramkrelidze, Volume 19, Springer-Verlag, Berlin-Heidelberg, 1992. 